\title{
¿Es hora de usar indicadores refinados para estudiar la fecundidad en América Latina?*
}

\author{
Wanda Cabella* \\ Ignacio Pardo***
}

El artículo discute la pertinencia de los llamados indicadores refinados de fecundidad para evaluar el nivel de la fecundidad en los países de América Latina. La principal ventaja de estos indicadores es su capacidad de identificar cuánto del cambio en la tasa global de fecundidad se debe a cambios en la intensidad y cuánto a cambios en el calendario. Los países de muy baja fecundidad están culminando un proceso de postergación de la maternidad que solo pudo ser descrito adecuadamente una vez que surgieron estos indicadores. A pesar de que en América Latina hay indicios de que en algunos países se comienza a procesar la postergación de la fecundidad, es aún muy limitada la capacidad de elaborar indicadores refinados y no abunda la discusión sobre sus virtudes, desventajas y potencialidades. Con la intención de sistematizar la acumulación al respecto y fomentar el debate, en este artículo se presentan las principales características de estas medidas y se discute cuáles indicadores serían los más adecuados para evaluar las tendencias de la fecundidad en la región y cómo podrían difundirse de modo de evitar equívocos en su interpretación. Nuestra principal conclusión es que a corto plazo resulta más urgente contar con indicadores de intensidad y calendario por orden de nacimiento que apelar a medidas sintéticas refinadas.

Palabras clave: Indicadores refinados de fecundidad. Posponement transition. América Latina. Uruguay.

\footnotetext{
* Este trabajo fue realizado fue realizado en el marco del proyecto de investigación La caída de la fecundidad en Uruguay (1996-2011). ¿Cuál es su verdadera dimensión?, que contó con el apoyo financiero de la Comisión Sectorial de Investigación Científica (CSIC) de la Universidad de la República, Uruguay. Los autores agradecen las contribuciones de la Editora de Rebep y de los tres evaluadores anónimos que revisaron el artículo.

** Programa de Población, Facultad de Ciencias Sociales, Universidad de la República, Montevideo, Uruguay (wanda. cabella@cienciassociales.edu.uy).

*** Programa de Población, Facultad de Ciencias Sociales, Universidad de la República, Montevideo, Uruguay (Ignacio. pardo@cienciassociales.edu.uy).
} 


\section{Introducción}

En los países cuya fecundidad ha llegado a etapas avanzadas de la Postponement Transition, la utilización de indicadores refinados permitió atemperar los problemas de la tasa global de fecundidad (TGF), interpretar mejor la evolución de la fecundidad en el contexto de postergación de los nacimientos y perfeccionar los supuestos de las proyecciones de población. Estos indicadores refinados complejizan la habitual medición por cohortes sintéticas, aislando los cambios de calendario que provocan el llamado efecto tempo y a menudo incorporando otros ajustes, como los cambios en la estructura por paridez de la población femenina. Dado que en algunos países de América Latina se observan síntomas de aplazamiento de la maternidad (CABELLA; PARDO, 2014; ESTEVE et al., 2012; ROSERO-BIXBY et al., 2009), es oportuno tratar el tema de los principales usos y fundamentos de estos indicadores, tomando en cuenta las controversias que generaron en los países que los usan intensamente.

El objetivo de este artículo es discutir la utilidad de los indicadores refinados de fecundidad para los países de América Latina -en el contexto más amplio de la necesidad de indicadores de fecundidad apropiados para la región-,que permitan dar cuenta de las nuevas pautas del comportamiento reproductivo tanto como difundir adecuadamente las tendencias de la fecundidad.

En primer lugar, se reseña el contexto demográfico del surgimiento de estos indicadores y se discuten sus condiciones de aplicación y la pertinencia de su uso. Apenas a modo de ilustración empírica se presenta brevemente una batería de indicadores refinados de fecundidad para el caso uruguayo. Luego se presenta la evolución reciente de la TGF y la edad media a la maternidad (EMM) de varios países latinoamericanos, con el objeto de introducir el debate sobre el eventual uso de indicadores refinados. Finalmente, se discuten las implicaciones del uso de estos indicadores en términos de la relación entre fecundidad de cohorte y de período, tomando los principales ejes de la discusión que tuvo lugar en los países que están culminando la Postponement Transition.

\section{El surgimiento de las medidas refinadas en los países de fecundidad muy baja}

En este artículo se tomarán en cuenta los indicadores refinados de fecundidad más habituales: aquellos que ajustan el nivel de la fecundidad de momento por el efecto tempo, aunque también son conocidas otras variantes, como las que agregan el ajuste por los cambios en la estructura de paridez de la población femenina. La principal ventaja de estas medidas es su capacidad de identificar cuánto del cambio en la TGF se debe a cambios en la intensidad y cuánto a cambios en el calendario.

Los indicadores refinados fueron cruciales para describir retrospectivamente la fecundidad de varios países entre inicios de la década del setenta y los primeros años del siglo XXI, cuando una caída drástica de la TGF fue seguida de un sorpresivo repunte en casi todos los casos. La reversión de la tendencia respondió sobre todo a la desaceleración 
del aumento en la edad de los nacimientos (BONGAARTS; SOBOTKA, 2012; MYRSKYLÄ et al., 2009). Es decir que un indicador coyuntural de intensidad de la fecundidad se había modificado principalmente a impulso de cambios de calendario.

El fenómeno demográfico que motivó esos cambios es hoy bien conocido y se lo ha denominado Postponement Transition, una sucesión de varios años de aumento en la EMM seguido por un período de desaceleración que conduce a una fase final en la que el indicador deja de aumentar y se estabiliza en valores altos (en torno a los treinta años o más para el primer nacimiento). En los países europeos de transición demográfica muy avanzada, el proceso se inició en la década del setenta, se intensificó durante varios años hasta fines de los años primeros años del siglo XXI (KOHLER et al., 2002), a menudo hasta que la TGF alcanzara niveles muy bajos (lowest-low ${ }^{1}$ ), y está llegando a su fin en el presente. Fue similar la experiencia de algunos países asiáticos, con ejemplos emblemáticos como Japón, Corea del Sur y Singapur.

El concepto de Postponement Transition permitió poner en perspectiva la caída del nivel de la fecundidad de período, que no había implicado disminuciones de similar magnitud en la fecundidad de las cohortes (SOBOTKA et al., 2011). Así, el aumento del nivel de fecundidad de período pasó a ser conocido en la bibliografía como el rebote (rebound) de la TGF (BONGAARTS; SOBOTKA, 2012) y su explicación quedó asociada fundamentalmente a los supuestos de construcción del indicador. En el Gráfico 1 se pueden observar varios ejemplos ilustrativos del fenómeno en países europeos y Japón.

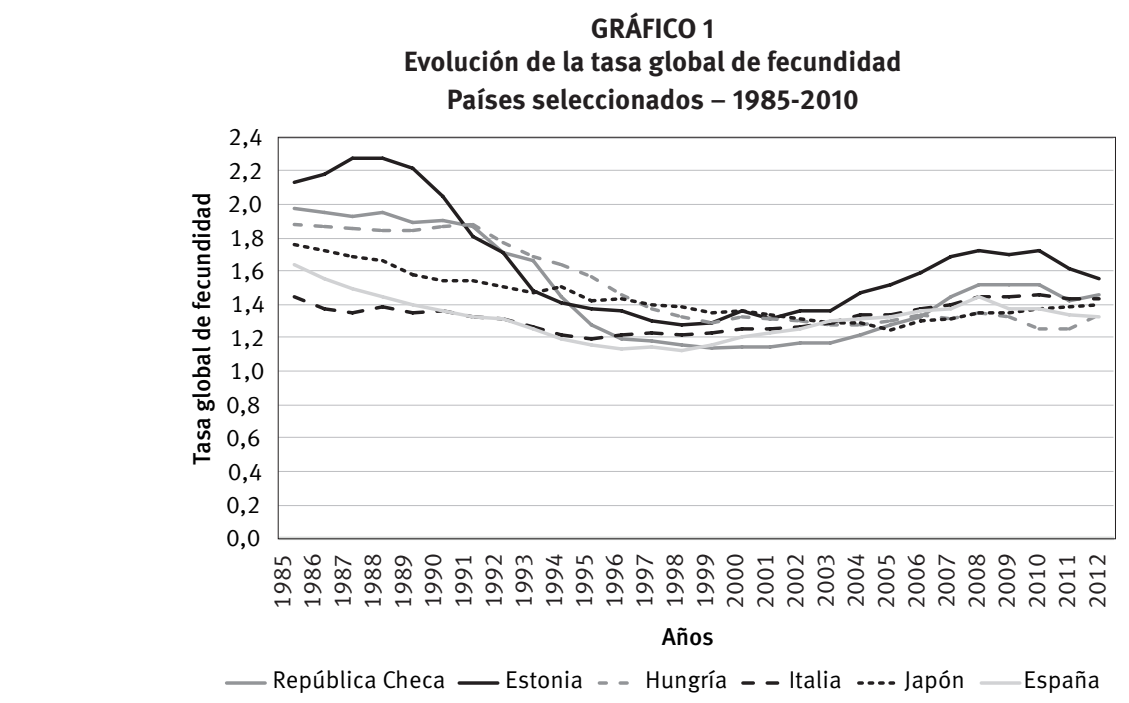

Fuente: Human Fertility Database, disponible en 〈http://www.humanfertility.org/〉.

\footnotetext{
${ }^{1}$ Se asume el umbral de 1,5 hijos por mujer para hablar de fecundidad muy baja, aunque se utiliza también el umbral de 1,3 hijos por mujer. Con ese umbral, más exigente, un número nada despreciable de países europeos llegó a niveles muy bajos.
} 
En una primera instancia, la explicación de este rebote de la TGF no era evidente. Podía deberse a un aumento efectivo del número de hijos (modificación del quantum), reflejar cambios en la evolución de la EMM (modificación del tempo) o ser el resultado de una combinación de ambos fenómenos, como finalmente fue el caso, pero con una influencia muy considerable del efecto tempo (BONGAARTS; SOBOTKA, 2012; SOBOTKA, 2001). Esto puso en primer plano el debate acerca de las medidas sintéticas de fecundidad y sus versiones ajustadas.

\section{Un repaso de los indicadores refinados de fecundidad, su cálculo y sus principales problemas}

La dificultad para deslindar las posibles fuentes de variación de la TGF dejó en exposición su principal problema: su incapacidad para diferenciar en qué medida su variación responde a cambios de la intensidad de momento -que es lo que pretende medir-, y en qué medida responde a cambios en el calendario de la cohorte sintética, que es utilizada para medir la fecundidad de período. Dicho de otro modo, la postergación o el adelantamiento de los nacimientos pueden generar "distorsiones" (ya veremos por qué entre comillas), a través del llamado efecto tempo. Concretamente, cuando hay modificaciones en la EMM de un año al siguiente, la TGF convencional no permite evaluar si la modificación del nivel de la fecundidad, medido como el número de hijos que la cohorte sintética tendría al final de su vida reproductiva, está afectada por el cambio de calendario. Si hay aplazamiento en el calendario, la TGF subestimará el quantum de la fecundidad y, a la inversa, lo sobreestimará si se producen adelantamientos. La adecuación del uso de términos como "subestimación" o "sobreestimación" es objeto de controversia: se asume que se estima por encima o por debajo en relación con el valor futuro de la fecundidad completa de las cohortes, pero a los efectos de la medición puntual, la TGF cumple su función. Es decir, determina el nivel de la fecundidad de un año o período, sin importar el peso de los factores que contribuyen a modificarlo. Más adelante en el texto se volverá sobre este punto.

Se ha identificado otra potencial fuente de “distorsión", originada en el cambio en la composición de la población femenina según paridez, que modifica los denominadores de las tasas de un año determinado, dados los cambios en la fecundidad de años anteriores. A modo de ejemplo, un descenso sistemático en los primeros nacimientos genera un aumento progresivo de la proporción de mujeres con paridez cero, por lo que la población expuesta a tener su primer hijo es mayor a medida que en los años pasados se haya reducido el número de mujeres que se convirtieron en madres. Esta "distorsión" adicional se denomina efecto paridez y suele tener una magnitud mucho menor que el efecto tempo en la variación de la intensidad de la fecundidad de período.

Como se dijo, la necesidad de aislar la medición de la intensidad de momento de otros fenómenos, fundamentalmente del efecto tempo, condujo al desarrollo de nuevas 
medidas sintéticas de fecundidad. La mayoría de estas medidas surgió hacia fines de la década del noventa y se basó en el trabajo pionero de Ryder $(1956,1959,1964)$. Es el caso de Bongaarts y Sobotka (2012), Ní Bhrolcháin (2011), Luy (2010), Luy y Pöstch (2010), McDonald y Kippen (2009), Kohler y Ortega (2007, 2002a, 2002b), Corr y Kippen (2006), Bongaarts y Feeney $(2006,1998)$, Kippen (2003) y Rallu y Toulemon (1993), entre otros. Kohler y Ortega (2004, p. 60) resumen de forma intuitiva la motivación de las medidas ajustadas por tempo:

The number of births occurring in a year that is characterized by tempo changes [...] differs from the number of births that would have occurred in this year in the absence of the timing change. Correcting for these "missing" or "excess" births in a calendar year is therefore the basic idea behind the adjustment for tempo effects (or tempo distortions): tempo effects are defined as the proportional change in fertility rates and period fertility measures that are due to shifts in the timing of fertility. These tempo distortions affect incidence rates, childbearing intensities, and all derived measures such as TFR, etc.

Hay consenso en que Bongaarts y Feeney (1998) contribuyeron decisivamente al proponer que las medidas ajustadas definieran el componente de quantum de la fecundidad como aquel que se hubiera observado en ausencia de cambios en el calendario de la maternidad durante el período en que se mide la tasa global de fecundidad (BONGAARTS; FEENEY, 1998, p. 272). ${ }^{2}$ La idea básica es estimar el efecto tempo y obtener un valor diferente de la TFG en caso de que ese efecto sea diferente de cero. La medida propuesta por estos autores se conoce como TFG ajustada, adjTFR, TFR', o TFR*. ${ }^{3}$ En este trabajo se sigue la notación utilizada por Bongaarts y Sobotka (2012), quienes distinguen las medidas ajustadas por efecto tempo con un asterisco (para el caso, TFR ${ }^{\star}$ ). El ajuste consiste en dividir la TGF por un indicador de cambio en la EMM (véase en tabla 1 el modo de cálculo de la TFR*).

En los años que siguieron la TFR* fue objeto de críticas y reformulaciones. Una de las críticas que se le hizo al indicador es que se basaba en el supuesto de forma constante (constant shape assumption), es decir que el indicador reposa sobre el supuesto de que los cambios en el tempo son idénticos a todas las edades de las mujeres en cada período (KOHLER; PHILIPOV, 2001). Aunque hay quien lo encuentra demasiado restrictivo (INABA, 2003; KIM; SCHOEN, 2000), el supuesto ha demostrado cumplirse en las poblaciones con baja fecundidad (BONGAARTS; SOBOTKA, 2012; BONGAARTS; FEENEY, 2010). Por otro lado, el ya mencionado efecto paridez condujo a la elaboración de indicadores construidos con tasas del primer tipo (condicionales) para sustituir a las tasas de segundo tipo (incondicionales) utilizadas en la TGF y en la TFR*. En las tasas del primer tipo,

\footnotetext{
${ }^{2}$ Más estrictamente, con la TFR* se observa "what would have been observed in a year t if the pattern of age-specific fertility rates (for each birth order)/death rates (i.e., shape and location at the age axes) had been constant during the whole year and only the values of the rates had been change" (LUY, 2011, p. 442).

${ }^{3}$ En este trabajo se usan los términos en inglés a falta de traducciones de uso corriente al español de las siglas de los indicadores ajustados por tempo. Esta decisión facilita la notación y por ende la lectura del texto. Solo se mantiene el uso de la tasa global de fecundidad (TGF), el equivalente en castellano a Total Fertility Rate (TFR).
} 
el denominador está compuesto por la población de cierta edad expuesta al riesgo de experimentar el evento y no por toda la población comprendida en ese grupo de edad. Por ejemplo, las mujeres de cierta edad expuestas a experimentar el nacimiento de su primer hijo son exclusivamente las mujeres de esas edades y de paridez cero, es decir, aquellas que aún no han tenido hijos.

Adicionalmente, se desarrollaron las propuestas de la TFRp (la “p” refiere a paridez) y su versión ajustada por tempo, TFRp*. Estas medidas utilizan tasas de fecundidad del primer tipo y los nacimientos de cada orden son tratados como eventos separados, no repetibles. De esta manera, controlan las “distorsiones” que trae consigo el cambio en la distribución de las mujeres por paridez, refinando el denominador (BONGAARTS; FEENEY, 2006). ${ }^{4}$ Otras medidas desarrolladas con objetivos similares son la PATFR (Parityand age-adjusted TFR) y su versión ajustada por tempo (llamada PATFR* en Bongaarts y Sobotka (2012) y PF en su versión original) desarrolladas por Kohler y Ortega (2002a) y basadas en tablas de fecundidad. Estas medidas se basan en los trabajos de Rallu y Toulemon (1994) y Park (1976), y desarrolladas en mayor profundidad en Kohler y Ortega (2002a, 2002b, 2004) y en Kohler y Philipov (2001).

En el Cuadro 1 figuran en detalle las características de las principales medidas ajustadas: TFR*, TFRp* y PATFR*.

De haberse desarrollado antes los indicadores ajustados, es posible que algunas de las alarmas encendidas por la muy baja fecundidad de los países europeos se hubiesen matizado. El repunte de la TGF mostró que de no haber existido una fuerte presión a la baja ejercida por el efecto tempo, la caída a niveles tan bajos no hubiera sido posible (MYRSKILÄ et al., 2013). Actualmente, para la comunidad demográfica de estos países es impensable evaluar la magnitud de los cambios de la fecundidad y prever su evolución futura sin considerar el efecto tempo en las medidas sintéticas. La distinción quantum/ tempo para el análisis de la fecundidad de período (así como para otros fenómenos demográficos) se ha convertido en un aspecto tan central en el análisis demográfico que Luy (2010) ha hablado de un cambio de paradigma (paradigm shift) asociado a esta distinción. Cabe preguntarse si ha llegado el momento de que la comunidad de demógrafos latinoamericanos incorpore una lógica similar para el análisis de las poblaciones de la región, que muestran una dinámica de cambio en intensidad y calendario diferente a la del contexto de surgimiento de los indicadores refinados. A continuación se muestra el caso particular de Uruguay, que tuvo una evolución específica dentro del contexto de países latinoamericanos.

\footnotetext{
4 Yamaguchi y Beppu (2004) han trabajado en una medida muy similar.
} 
CUADRO 1

Descripción de los indicadores refinados de fecundidad más usados

\begin{tabular}{|c|c|c|c|}
\hline & $\begin{array}{l}\text { Medidas ajustadas } \\
\text { por tempo }\end{array}$ & & $\begin{array}{l}\text { didas ajustadas } \\
\text { tempo y paridez }\end{array}$ \\
\hline \multirow{2}{*}{$\begin{array}{l}\text { Sigla y } \\
\text { nombre } \\
\text { original }\end{array}$} & TFR* & PATFR* & TFRp* \\
\hline & $\begin{array}{l}\text { Tempo adjusted Total Fertility } \\
\text { Rate }\end{array}$ & $\begin{array}{l}\text { Tempo-adjusted Parity- } \\
\text { and Age Total Fertility Rate }\end{array}$ & $\begin{array}{l}\text { Tempo and parity-adjusted total fertility } \\
\text { rate }\end{array}$ \\
\hline \multirow{2}{*}{$\begin{array}{l}\text { Sigla y } \\
\text { nombre } \\
\text { propuestos } \\
\text { en español }\end{array}$} & TGF* & $\mathrm{TGFp}^{*} \_\mathrm{K} \& \mathrm{O}$ & TGFp*_B\&F \\
\hline & $\begin{array}{l}\text { Tasa global de fecundidad } \\
\text { ajustada por tempo }\end{array}$ & $\begin{array}{l}\text { Tasa global de fecundidad } \\
\text { ajustada por paridez y } \\
\text { tempo, variante Kohler y } \\
\text { Ortega }\end{array}$ & $\begin{array}{l}\text { Tasa global de fecundidad ajustada por } \\
\text { paridez y tempo, variante Boongarts y } \\
\text { Feeney }\end{array}$ \\
\hline $\begin{array}{l}\text { Datos } \\
\text { numerador }\end{array}$ & $\begin{array}{l}\text { Nacimientos según edad } \\
\text { de la madre y orden de } \\
\text { nacimiento }\end{array}$ & $\begin{array}{l}\text { Nacimientos según edad } \\
\text { de la madre y orden de } \\
\text { nacimiento }\end{array}$ & $\begin{array}{l}\text { Nacimientos según edad de la madre y } \\
\text { orden de nacimiento }\end{array}$ \\
\hline $\begin{array}{l}\text { Datos } \\
\text { denominador }\end{array}$ & Mujeres según edad & $\begin{array}{l}\text { Mujeres según edad y } \\
\text { paridez }\end{array}$ & Mujeres según edad y paridez \\
\hline $\begin{array}{l}\text { Modo de } \\
\text { cálculo }\end{array}$ & $\begin{array}{c}\mathrm{t}=\text { año; } \mathrm{i}=\text { orden de } \\
\text { nacimiento; EMM }=\text { Edad } \\
\text { Media a la Maternidad } \\
\mathrm{TFR}^{\star}=\sum \mathrm{TFR}_{i}^{\star} \\
\mathrm{TFR}_{i}^{\star}=\frac{\operatorname{TFR}_{i}(t)}{1-\mathrm{r}_{i}(t)}\end{array}$ & $\begin{array}{l}\text { Su cálculo se realiza } \\
\text { a partir de tablas } \\
\text { de fecundidad y los } \\
\text { denominadores de las } \\
\text { tasas son en este caso } \\
\text { la población femenina } \\
\text { de edad x y paridez i-1. } \\
\text { Al igual que la TFRp, } \\
\text { neutraliza el efecto de los } \\
\text { cambios en la distribución } \\
\text { de las mujeres por paridez. } \\
\text { El ajuste obtenido en la } \\
\text { PATFR* requiere cálculos } \\
\text { más complejos, pues no } \\
\text { solo involucra la edad } \\
\text { media a los nacimientos } \\
\text { sino también su varianza. }\end{array}$ & $\begin{array}{l}\operatorname{TFRp}(t)^{\star}=\sum_{i} \operatorname{TFRp}(\mathrm{t}, \mathrm{i}) \sum_{i}\{1 \exp \\
\quad\left[-\sum_{x}(\mathrm{p}(\mathrm{a}, \mathrm{t}, 1-\mathrm{r}(1,1))]\right\} \\
\mathrm{a}=\text { edad; } \mathrm{t}=\text { año; } \mathrm{i}=\text { orden } \\
\mathrm{p}(\mathrm{x}, \mathrm{t}, \mathrm{i})=\text { tasas de fecundidad del } \\
\text { primer tipo (condicionales) con los } \\
\text { nacimientos de cada orden tratados } \\
\text { como eventos separados no repetibles } \\
\text { Los denominadores de las tasas de } \\
\text { orden i incluyen a todas las mujeres de } \\
\text { paridez } \mathrm{j} \text { tal que } \mathrm{j}<\mathrm{i} \text {, como se dijo más } \\
\text { arriba }\end{array}$ \\
\hline \multirow[t]{2}{*}{$\begin{array}{l}\text { Documentos } \\
\text { de referencia } \\
\text { del indicador } \\
\text { o similares }\end{array}$} & Bongaarts y Feeney (1998) & $\begin{array}{l}\text { Yamaguchi y Beppu } \\
\text { (2004); Kohler y Ortega } \\
\text { (2002); Rallu y Toulemon } \\
\text { (1994), Park (1976) }\end{array}$ & Bongaarts y Feeney (2006) \\
\hline & Bongaarts y Sobotka (2012) & & \\
\hline
\end{tabular}

Nota: Existen indicadores similares a PATFR*, pero ajustados por duración intergenésica (PADTFR) (MIRANDA-RIBEIRO et al., 2013; NÍ BHROLCHAIN, 1992).

\section{La evolución de los indicadores refinados de fecundidad en Uruguay}

Uruguay es un país de fecundidad históricamente baja (PELLEGRINO, 2010; CHACKIEL; SCHKOLNIK, 1992), que desde 1996 experimentó una nueva etapa de descenso. Durante esta caída, la TGF cayó por debajo del nivel de reemplazo (de 2,5 en 1996 a 1,9 en 2011), alcanzando niveles similares a los de otros países de la región. Para discutir los puntos abordados en este artículo, observamos su evolución y agregamos la versión ajustada por tempo (TFR*) y la TFRp*, ajustada por tempo y paridez (Gráfico 2).

El efecto tempo, es decir, la diferencia entre tasa global de fecundidad y TFR*, es mayor a cero en algunos años entre 1996 y 2011, pero no se observa una tendencia al aplazamiento que sea estable a lo largo de todo el período. Concretamente, se observa 
un aumento significativo de las medidas ajustadas entre 2002 y 2003 , coincidentes con la crisis económica que vivió el país a inicios de esa década. De 2004 en adelante, ambas medidas ajustadas presentan niveles más cercanos a la tasa global convencional. Es posible que en respuesta a las condiciones económicas adversas, las parejas hayan aplazado los nacimientos por dos o tres años, pero en tal caso el fenómeno culminó muy rápidamente. Una mirada que abarque los quince años debería destacar que entre 1996 y 2011 no hubo modificaciones de entidad en la EMM que generasen valores superiores de la TFR* en relación con la TFR. En suma, el descenso registrado entre 1996 y 2011 no es esencialmente resultado de la acción del efecto tempo, sino del declive del quantum de la fecundidad de período en esos años.

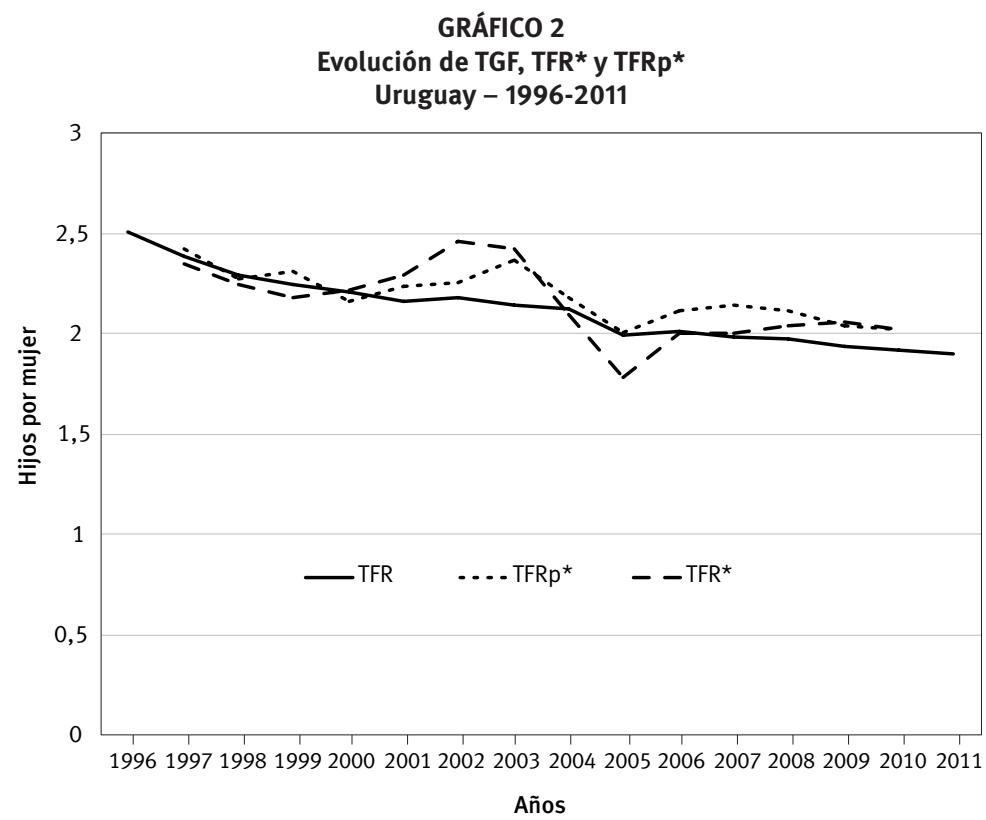

Fuente: Ministerio de Salud Pública (MSP). Microdatos del Registro de Nacimientos; Instituto Nacional de Estadística INE). Proyecciones de Población (Revisión 2013).

De todos modos, ¿cómo interpretar estos movimientos abruptos de los indicadores ajustados en los primeros años del siglo XXI? En este caso, una interpretación automática de la TFR* implicaría sostener que de no haber aumentado la EMM, la TGF habría mostrado un extraordinario repunte en esos dos años. Pero ¿cuán sensata es esa lectura? Asumir que de no haber existido aumento en la EMM, la TGF se habría situado en torno a 2,5 hijos durante los dos peores años de la crisis, aunque sea estrictamente lo que sugiere el indicador, no parece una opción recomendable. Cuando la TFR* presenta oscilaciones bruscas es más sensato no considerar el valor contrafactual de su interpretación, más adecuado para un contexto de postergación paulatina en poblaciones de fecundidad baja.

En definitiva, ¿no hubo postergación de los nacimientos? El escaso cambio de la EMM en todo el período puede tomarse como una señal de que el calendario de la fecundidad 
no se ha modificado, aunque lo cierto es que hubo cambios. Fueron casi imperceptibles cuando se observan todos los órdenes juntos, pero visibles si se observa la edad media de los primeros y segundos nacimientos, en los que hubo un aumento sostenido, indicativo del posible comienzo de la Postponement Transition en el país (NATHAN; PARDO; CABELLA, 2016). Las medidas sintéticas no registran el proceso porque sucede al mismo tiempo que las mujeres detienen su fecundidad en parideces menores -lo que reduce los nacimientos de órdenes superiores, que ocurren a edades más avanzadas, y por tanto impulsa al descenso de la EMM-. Ambos fenómenos se compensan y como resultado no hay aumento importante de la EMM total; por eso la TFR* tampoco es una medida elocuente en ausencia de aumentos sistemáticos de la EMM.

\section{Las medidas de fecundidad y el contexto de América Latina y el Caribe}

¿Cómo se han tratado estos temas en la región? El uso de medidas ajustadas por tempo es muy incipiente, pero se han realizado algunos ejercicios. Además del caso uruguayo, concretamente, existen análisis recientes de la fecundidad brasileña (MIRANDA-RIBEIRO et al., 2013; MIRANDA-RIBEIRO et al., 2016) que utilizan datos censales de los últimos treinta años para reconstruir historias de nacimientos y elaborar una gran variedad de indicadores refinados, a menudo a partir de las sugerencias de Kohler y Ortega (2007) en torno al uso de tablas de fecundidad. Así es posible controlar los efectos tempo, paridez y en algún caso también el efecto duración (desde el nacimiento anterior). La conclusión suele ser que, además de un efecto paridez positivo, existió en la TGF brasileña un efecto tempo negativo (dado por el efecto compensador de la caída de la fecundidad en los órdenes altos), que en los años más recientes se volvió levemente positivo, como sucede en los países de fecundidad baja que inician el proceso de aplazamiento de los nacimientos.

Unos años antes, también apelando a censos y a reconstrucción de historias de nacimientos, Miranda-Ribeiro et al. (2008) contrastaron la evolución de la TGF brasileña con la mexicana (1985-2000). Los indicadores refinados revelaron que el caso mexicano no es idéntico al brasileño pero se asemeja. Existe un efecto paridez, al igual que en Brasil, y la evolución del efecto tempo también es coincidente: desde 1985 es negativo, pero comienza a acercarse a cero al comenzar la década del noventa, para mantenerse en valores muy cercanos a ese nivel hasta el año 2000, dado el menor ritmo de cambio de la EMM.

Los resultados obtenidos para estos países no sorprenden si se considera que la caída reciente de la fecundidad en América Latina hacia niveles bajos estuvo básicamente influenciada por la reducción de los nacimientos de órdenes superiores, sin un descenso abrupto de los niveles de fecundidad adolescente, lo que condujo a un rejuvenecimiento de la EMM. Solo muy recientemente se observan indicios de un desplazamiento de la EMM hacia edades más tardías en algunos países (Brasil, Chile, Colombia, Puerto Rico, Uruguay, por ejemplo) (Gráfico 3). 
El descenso de la tasa global de fecundidad en los países latinoamericanos desde el quinquenio 1990-1995 no tiene excepciones. En general, sucedió por la reducción de las tasas en las edades centrales de la reproducción (20 a 29 años) y hubiera sido más fuerte de no mediar la persistencia de tasas de fecundidad adolescente relativamente altas (CEPAL, 2011; RODRÍGUEZ; CAVENAGHI, 2014; CABELLA; PARDO, 2014; RODRÍGUEZ, 2015). Como resultado, hay una considerable cantidad de países en el entorno del umbral de reemplazo. En segundo lugar, la EMM tiende a permanecer estable o a disminuir, en la línea de lo observado más en detalle para Brasil y México hasta hace relativamente poco tiempo. Estos patrones tienen consecuencias a la hora de pensar en los indicadores más adecuados para resumir la intensidad de la fecundidad de los países: el contexto en el que surgieron los indicadores ajustados (el rebote de la fecundidad en los países europeos que tenían niveles bajo el remplazo desde hacía varios años) difiere mucho del contexto actual de nuestra región. Básicamente, porque la EMM de todos los órdenes de nacimiento aún no sube de forma importante; en algún caso porque no ha comenzado la Postponement Transition, en otros por los efectos de compensación que se mencionaban unos párrafos atrás.

GRÁFICO 3

Evolución de la tasa global de fecundidad y la edad media a la maternidad Subregiones y países seleccionados - 1990-2015
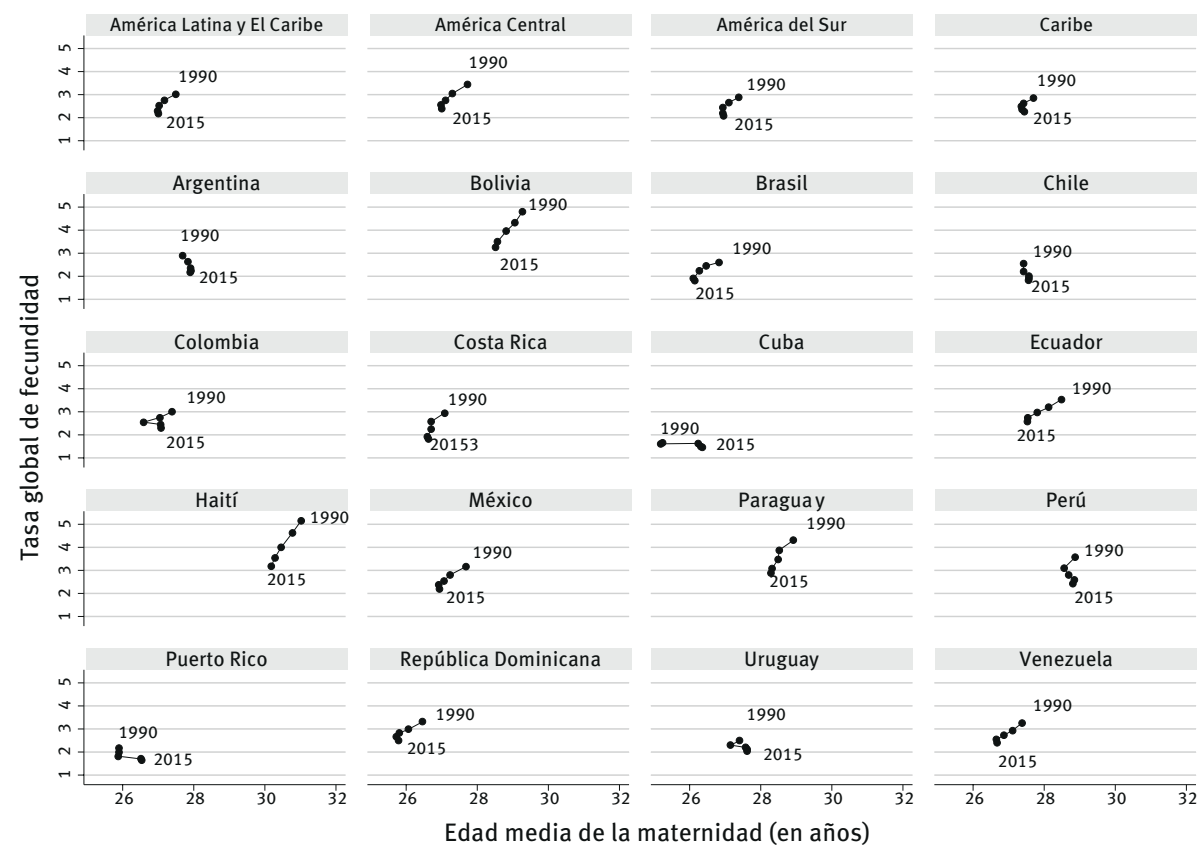

Fuente: Cabella y Pardo (2014, p. 18). Reimpreso con autorización.

En todo caso, en los países de la región que aún no tienen niveles de fecundidad muy bajos (casi todos, con excepción de los caribeños), no es claro que las tendencias generales de intensidad y calendario vayan a evolucionar exactamente como lo hicieron 
en los países europeos. De utilizarse la trayectoria de los modelos estilizados habituales, ${ }^{5}$ se diría que muchos de los países latinoamericanos están atravesando una etapa inicial del descenso, en la que no son esperables cambios en el calendario, mientras otros comienzan una segunda etapa, en la que el aplazamiento comienza a ser visible. Así, los indicadores sintéticos ajustados por tempo no serían de mayor utilidad en el presente pero sí podrían serlo en el futuro. Sin embargo, es necesario observar las dinámicas específicas por orden de nacimiento para conocer cómo está compuesto el cambio en la fecundidad de cada país, lo que requiere datos de calidad y acumulación creciente en el estudio de la fecundidad por orden.

Lamentablemente, la investigación sobre las tendencias y explicaciones de los patrones de la fecundidad en América Latina es poco abundante en los últimos tiempos, si bien se han producido publicaciones relevantes en torno al tema (CABELLA; PARDO, 2014; CAVENAGHI, 2009; CAVENAGHI; ALVES, 2011, 2016; MIRANDA-RIBEIRO et al., 2016; NATHAN; PARDO; CABELLA, 2016; RIOS NETO; GUIMARÃES, 2014; ROSERO-BIXBY; CASTRO-MARTIN; MARTINGARCIA, 2009, para nombrar las más recientes). También es escasa la presencia de la fecundidad en los encuentros científicos regionales: en los últimos tres congresos de la Asociación Latinoamericana de Población (ALAP), la fecundidad fue tema de solo una de 63 sesiones regulares (2012), 2 de 56 (2014) y 5 de 70 (2016), ${ }^{6}$ respectivamente. Quizá el decaimiento en el interés se deba a que estamos en un interregno entre dos circunstancias que en diferentes momentos históricos han sido objeto de preocupación de los demógrafos: la vieja preocupación por la alta fecundidad ya desapareció y no se ha instalado aún la preocupación por el "invierno demográfico" que las miradas catastrofistas promueven en los países de fecundidad muy baja (DUMONT, 2008).

Vista de otro modo, la relativa ausencia del tema puede vincularse al rezago en el cambio de enfoque que viene procesándose en otras regiones, desde la clásica mirada centrada en la relación entre fecundidad, reproducción de las poblaciones y crecimiento demográfico (según la cual la fecundidad demasiado alta o demasiado baja es más importante que cualquier otra cosa), hacia la descripción y explicación del comportamiento reproductivo per se. Este cambio de paradigma en la mirada teórica sobre el tema es concomitante con el que se ha observado en términos de medición a partir del uso de indicadores refinados, en particular aquellos que se detienen en el análisis de la dinámica de cambio de la fecundidad por orden de nacimiento, para observar comportamientos cada vez más específicos.

¿Qué vida tiene por delante la tasa global de fecundidad en este contexto? Los indicadores sintéticos convencionales son una herramienta de gran potencial comparativo.

\footnotetext{
${ }_{5}^{5}$ Lesthaeghe y Willems (1999) describen el descenso de la fecundidad como un proceso que se produce en tres etapas. Una primera, en la que el descenso es básicamente de nivel y el calendario permanece poco cambiado; una segunda que da comienzo a la postergación del calendario, con la fecundidad en niveles bajos, y una tercera, en la que la postergación termina y la TGF aumenta por efecto del fin de la postergación, aunque sin recuperar del todo los niveles del pasado.

${ }^{6}$ Datos extraídos de los respectivos sitios de ALAP donde se encuentran depositados los programas académicos de los congresos: 〈http://www.alapop.org/alap/〉.
} 
Dado el comportamiento reproductivo de las poblaciones de fecundidad baja, como lo serán de modo creciente las latinoamericanas, se puede considerar a la TGF como una medida resumen aún de gran utilidad, sobre todo si surge de las estadísticas vitales de los países: brinda un panorama general de utilidad y es más confiable que los indicadores basados en información censal (CAVENAGHI; ALVES, 2016). No obstante, si el incipiente escenario de aplazamiento se desarrolla y consolida, será bueno complementarla con la TFR* $u$ otras medidas refinadas que permitan cumplir cabalmente con algunos de sus roles habituales.

Estas medidas refinadas pueden contribuir a visualizar problemas específicos, pero tampoco están libres de problemas; en definitiva, no son más que un ajuste de la TGF, a la que se incorporan supuestos adicionales. No hay forma de escapar a la necesidad de un conjunto más amplio de indicadores que incluya medidas por orden de nacimiento. Si bien cualquier indicador es un artificio, no es exagerado decir que la descripción más acabada del comportamiento real de las personas se logra a través de los indicadores de fecundidad por orden. Las personas toman decisiones respecto a tener o no un primer (o segundo, o tercer) hijo y respecto al momento que les resulta más adecuado para iniciar o continuar sus proyectos reproductivos. Como se ha mostrado para Uruguay y otros países de la región (NATHAN; PARDO; CABELLA, 2016; LIMA et al., 2015; CABELLA; PARDO, 2014; MIRANDA-RIBEIRO et al., 2013, 2016, 20087) y se ha señalado en este mismo artículo, lo habitual es que existan dinámicas por orden de nacimiento que compensen sus efectos a nivel agregado y se tornen invisibles a partir de las medidas sintéticas, lo que socava su utilidad como descripción del comportamiento reproductivo.

En ese escenario es necesario actualizar el conjunto de indicadores de fecundidad. Mientras la TGF es útil para reflejar procesos de cambio en la intensidad de largo plazo (típicamente, la reducción de valores altos a bajos) hay que ubicar adecuadamente las potencialidades de esta medida y evitar su frecuente reificación. Si se la presenta de forma aislada y aproblemática, como descripción exacta de la fecundidad del país, o como proxy perfecto de la fecundidad final de las cohortes, podemos estar exigiendo a esa medida más de lo que puede dar. Por eso, contar con la fecundidad y la EMM por orden no es un lujo de los investigadores sino una necesidad primaria para conocer el comportamiento reproductivo. Además, es especialmente necesario un mayor énfasis en las medidas por estrato social, ya que la heterogeneidad de comportamientos reproductivos es norma en los países latinoamericanos. Es probable que el patrón específico de la fecundidad latinoamericana en las próximas décadas esté dado por la combinación de niveles bajos y convergentes en la intensidad, por un lado, y comportamientos heterogéneos de calendario, por otro (CABELLA; PARDO, 2014; CAVENAGHI; ALVES, 2011).

Finalmente, esta heterogeneidad de calendario hará cada vez más necesarias otras medidas: las de dispersión, como el desvío estándar de la EMM de primer o segundo

\footnotetext{
${ }^{7}$ Trabajos como Miranda-Ribeiro et al. (2013), basados en datos censales, pueden presentar los habituales problemas de subregistro de nacimientos.
} 
orden, o aún más específicas, como las que identifican una distribución bimodal en el calendario de los primeros nacimientos. Este fenómeno fue observado originalmente en los países anglosajones y empieza a describirse en algunos países de la región (NATHAN; PARDO; CABELLA, 2016; LIMA et al., 2015; SULLIVAN, 2005).

\section{Comentarios finales}

Al igual que las convencionales, las medidas ajustadas no son más que una de las variantes de las medidas basadas en cohortes hipotéticas. De hecho, pueden ser vistas como una extensión del control de los efectos de composición de la población femenina por edad fértil que caracteriza a la TGF (LUY, 2010). No es ocioso repetir que el debate acerca de los indicadores ajustados debe centrarse en esos términos de utilidad o pertinencia. Las medidas convencionales o las ajustadas no son correctas o incorrectas en sí mismas: ambos tipos de medidas son apropiadas para ciertos fines y se ajustan pobremente a otros (BONGAARTS; FEENEY, 2010; GUILLOT, 2006). En el caso de la tasa global convencional, cada año calendario la cohorte ficticia conformada por las mujeres de todas las edades fértiles tiene efectivamente las tasas incondicionales específicas cuya suma genera el valor de esa medida; por ese motivo puede ser necesario escribir con comillas que esa medida provoca “distorsiones". Es su interpretación habitual como proxy de la fecundidad de cohorte la que podría ambientar conclusiones equivocadas cuando la relación entre las tasas específicas por edad se modifica, generando efectos tempo y -ahora sí sin comillasdistorsionando su capacidad predictiva (GUILLOT, 2006; BONGAARTS; FEENEY, 2010, 2009; NÍ BROLCHAIN, 2010).

Lo problemático es que este uso como proxy se haya reificado, pues hay consenso en que ni los indicadores ajustados ni la TGF convencional, en tanto son medidas de período, pueden evaluar la fecundidad real de las cohortes (NÍ BHROLCHÁIN, 2010; BONGAARTS; SOBOTKA, 2012; BOONGARTS; FEENEY, 2009). Por eso hay quienes, en posición minoritaria, aconsejan desechar del todo ese uso y utilizar proyecciones propiamente dichas (NÍ BHROLCHÁIN, 2010). Lo que puede decirse es que en ciertos contextos la TFRp*, por ejemplo, muestra una importante correlación con la fecundidad completa de las cohortes (BONGAARTS; SOBOTKA, 2012).

El debate se encuadra en la ambivalencia de cualquier medición de este tipo. Por un lado, los indicadores de cohorte son los únicos que refieren a individuos reales, pero para conocer toda su trayectoria es necesario esperar a que culminen su vida reproductiva, por lo que se trata de indicadores que describen eventos del pasado. Por otro, las decisiones reproductivas y las decisiones de política pública se toman en el año calendario. Como recoge la expresión de Calot (1994), el mundo en que vivimos está en “modo período" ("the world we live in is periodwise").

La provocativa pregunta de Sobotka y Lutz (2011), quienes titularon su artículo "Los engañosos mensajes de política de la TGF: ¿deberíamos dejar de usarla?” (“Misleading 
policy messages derived from the period TFR: should we stop using it?"), es un acierto en el contexto europeo. Sin embargo, el caso de Uruguay muestra que puede tener poco valor heurístico extender el uso de estas medidas allí donde no hay postergación sostenida y monótona de la EMM, a riesgo de caer en lecturas poco sensatas de los indicadores ajustados. Cuando existen grandes shocks que agregan o quitan nacimientos de forma impactante en períodos cortos, como puede suceder ante una crisis, la interpretación habitual de los indicadores ajustados como medida contrafáctica puede llevar a conclusiones poco razonables, peores que las "distorsiones" que pretenden corregir, lo que las menoscaba como herramienta de resumen de información demográfica.

La postergación sostenida no se observa aún en América Latina de una forma que pueda repercutir en la TGF, aunque esto probablemente sucederá cuando la enorme mayoría de los nacimientos sean de primer o segundo orden. Por ahora, el aumento en la EMM de esos órdenes está comenzando en ciertos estratos de la población, lo que reafirma el valor de los indicadores de fecundidad por orden y estrato social de los padres. Y vuelve a traer a escena el problema de la calidad de los datos. Sobre todo, la necesidad de ampliar el rango y la calidad de preguntas relevadas en las estadísticas vitales de fecundidad.

La ampliación del conjunto de indicadores que describen la fecundidad tiene asimismo interés para la divulgación de resultados y la generación de insumos para las políticas de población que pudieran generarse a partir de la información demográfica, en un contexto en el que la experiencia europea sirve de referencia para el diseño de políticas, pero que necesita de un importante esfuerzo de adaptación a las especificidades de nuestras poblaciones (PARDO; VARELA, 2013). Y mejorar la medición de la fecundidad de período permite prever mejor la fecundidad que acumularán las cohortes al llegar al fin de su vida reproductiva, ya que su uso como proxy, aun siendo imperfecto, tiene utilidad. Ante la intensificación de los cambios de calendario, es incluso posible que debamos mirar más allá de medidas como la TFR* e incorporar intentos más novedosos, como el de Goldstein y Cassidy (2014), cuya TFR† ajusta la TGF, pero identificando la postergación de las cohortes, que así puede estimarse distinguiéndola de la postergación de período.

Al terminar, cabe responder qué hacer con la tasa global de fecundidad: should we stop using it? La respuesta depende del contexto; dado lo visto en las grandes tendencias de intensidad y calendario en América Latina y el Caribe (Gráfico 3), podría pensarse que aún es un indicador razonable del nivel de la fecundidad de período para América Latina y el Caribe y que conserva su utilidad para ciertos fines, pero que ya no es suficiente para describir sustantivamente el heterogéneo comportamiento reproductivo de los países de la región. Tampoco lo son las medidas ajustadas, consideradas de forma aislada. Para esa tarea, aunque las medidas sintéticas siempre tendrán utilidad como resumen, son necesarias herramientas más específicas: sobre todo, indicadores de intensidad y calendario por orden de nacimientos y estrato social. 


\section{Referencias}

BONGAARTS, J.; FEENEY, G. When is a tempo effect a tempo distortion? Genus, v. 66, n. 2, p. 1-15, 2010.

The quantum and tempo of life cycle events. Vienna yearbook of population research. Vienna Institute of Demography of the Austrian Academy of Sciences, 2006. p. 115-151.

On the quantum and tempo of fertility. Population and Development Review, v. 24, n. 2, p. 271-291, 1998.

BONGAARTS, J.; SOBOTKA, T. A demographic explanation for the recent rise in European fertility. Population and Development Review, v. 38, n. 1, p. 83-120, 2012.

CABELLA, W.; PARDO, I. Hacia un régimen de baja fecundidad en América Latina y el Caribe, 19902015. En: CAVENAGHI, S.; CABELLA, W. Comportamiento reproductivo y fecundidad en América Latina: una agenda inconclusa. Rio de Janeiro: Alap, 2014. p. 13-31 (Serie e-investigaciones n. 3). Disponible en: 〈http://www.alapop.org/alap/Serie-E-Investigaciones/N3/Capitulo1_SerieEInvestigaciones_N3_ALAP3.pdf〉.

CALOT, G. Synthetic measures based on rates or on probabilities. Population: An English Selection, n. 6, p. 95-107, 1994.

CAVENAGHI, S. (Org.). Demographic transformations and inequalities in Latin America: historical trends and recent patterns. Rio de Janeiro: Alap, 2009.

CAVENAGHI, S.; ALVES, J. E. D. Qualidade das informações sobre fecundidade no Censo Demográfico de 2010. Revista Brasileira de Estudos de População, v. 33, n. 1, p. 189-205, 2016.

Diversity of childbearing behaviour in the context of below-replacement fertility in Brazil. New York: United Nations, Department of Economic and Social Affairs, 2011 (Population Division Expert Paper, n. 2011/8).

CEPAL. Anuario Estadístico de América Latina y el Caribe 2011. Santiago de Chile: Cepal, 2011. CORR, P.; KIPPEN, R. The case for parity and birth-order statistics. Australian and New Zealand Journal of Statistics, v. 48, n.2, p. 171-200, 2006.

CHACKIEL, J.; SCHKOLNIK, S. La transición de la fecundidad en América Latina. Notas de Población, n. 55, LC/DEM/G.124, 1992.

DUMONT, G-F. Les conséquences géopolitiques de 'l'hiver démographique' en Europe. Géostratégiques, n. 20, p. 20-46, 2008.

ESTEVE, A.; GARCÍA-ROMAN, J.; LESTHAEGHE, R.; LÓPEZ-GAY, A. The "second demographic transition" features in Latin America: the 2010 update. Barcelona: Centre d'Estudis Demografics, Universitat Autonoma de Barcelona, 2012 (Working paper).

GOLDSTEIN, J.; CASSIDY, T. A cohort model of fertility postponement, Demography, v. 51, n. 5, p. 1797-819, 2014.

GOLDSTEIN, J.; SOBOTKA, T.; JASILIONENE, A. The end of 'lowest-low' fertility? Population and Development Review, v. 35, n. 4, p. 663-699, 2009.

HUMAN FERTILITY DATABASE. Max Planck Institute for Demographic Research (Germany) and Vienna Institute of Demography (Austria). Disponible en: 〈www.humanfertility.org〉. Acceso: 9 nov. 2016.

INABA, H. Resolving a confusion in the Bongaarts and Feeney's tempo-adjusted total fertility rate. Jinkogaku Kenkyu (The Journal of Population Studies), n. 32, p. 1-7, 2003. 
KIM, Y. J.; SCHOEN, R. On the quantum and tempo of fertility: limits to the Bongaarts-Feeney adjustment. Population Development Review, v. 26, n. 3, p. 554-559, 2000.

KOHLER, H. P.; BILLARI, F. C.; ORTEGA, J. A. The emergence of lowest-low fertility in Europe during the 1990s. Population Development Review, v. 28, n. 4, p. 641-680, 2002.

KOHLER, H.-P.; ORTEGA, J. A. Old insights and new approaches: fertility analysis and tempo adjustment in the age-parity model. Vienna yearbook of population research. Vienna Institute of Demography of the Austrian Academy of Sciences, 2004. p. 55-87.

KOHLER, H. P.; PHILIPOV, D. Variance effects in the Bongaarts-Feeney formula. Demography, n. 38, p. 1-16, 2001.

Tempo-adjusted period parity progression measures: assessing the implications of delayed childbearing for cohort fertility in Sweden, the Netherlands and Spain. Demographic Research, v. 6, article 7, p. 145-190, 2002a.

Tempo-adjusted period parity progression measures, fertility postponement and completed cohort fertility. Demographic Research, v. 6, article 6, p. 92-144, 2002b.

LESTHAEGHE, R.; WILLEMS, P. Is low fertility a temporary phenomenon in the European Union? Population and Development Review, v. 25, n. 2, p. 211-228, 1999.

LIMA, E.; ZEMAN, K.; CASTRO, R.; NATHAN, M.; SOBOTKA, T. Bimodal age-specific fertility profiles in Latin America: the case of Chile and Uruguay. En: ANUAL MEETING OF POPULATION ASSOCIATION OF AMERICA. Anais... San Diego, 2015.

LUY, M. Paradigm shift in demography? Editorial to the special issue "tempo effects in demographic period indicators. Comparative Population Studies -Zeitschrift für Bevölkerungswissenschaft, v. 35, n. 3, p. 409-414, 2010.

Tempo effects and their relevance in demographic analysis. Comp. Popul Stud, n. 35, p. 415-446, 2011.

LUY, M.; PÖTZSCH, O. Estimates of the tempo-adjusted total fertility rate in Western and Eastern Germany, 1955-2008. Comparative Population Studies -Zeitschrift für Bevölkerungswissenschaft, v. 35, n. 3, p. 605-636, 2010.

MYRSKYLÄ, M.; KOHLER, H. P.; BILLARI, F. Advances in development reverse fertility declines. Nature, n. 460, p. 741-743, 2009.

MIRANDA-RIBEIRO, A.; RIOS-NETO, A.; CARVALHO, J. A. M. Efeitos tempo, parturição e quantum no Brasil: indicadores de período e evidências empíricas. Revista Brasileira de Estudos de População, v. 30, n. 1, p. 145-170, 2013.

MIRANDA-RIBEIRO, A.; RIOS-NETO, E.;ORTEGA, J. A. Declínio da fecundidade no Brasil e México e o nível de reposição: efeitos tempo, parturição e quantum. En: WONG, L. (Ed.). Población y salud sexual y reproductiva en América Latina. Rio de Janeiro: Alap, 2008. p. 81-100.

MIRANDA-RIBEIRO, A.; KOHLER, H.-P.; RIOS-NETO, E.; GARCIA, R. A. Postergação ou antecipação? Uma análise do efeito tempo sobre a fecundidade brasileira nas últimas décadas. En: VII CONGRESO DE LA ASOCIACIÓN LATINOAMERICANA DE POBLACIÓN E XX ENCONTRO NACIONAL DE ESTUDOS POPULACIONAIS. Anais... Foz do Iguaçu: Alap/Abep, 2016.

NATHAN, M.; PARDO, I.; CABELLA, W. Diverging patterns of fertility decline in Uruguay. Demographic Research, v. 34, article 20, p. 563-586, 2016.

El descenso de la fecundidad en Uruguay según el orden de nacimiento (1996-2011). En: VI CONGRESO DE ALAP. Anais... Lima, Perú, 2014. 
NÍ BHROLCHÁIN, M. Tempo and the TFR. Demography, v. 48, n. 3, p. 841-861, 2011. Period paramount? A critique to the cohort approach to fertility. Population and Development Review, v. 18, n. 4, p. 599-629, 1992.

ORTEGA, J. A.; KOHLER, H.-P. Medición de la baja fecundidad: repensando los métodos demográficos. Estudios Demográficos y Urbanos, v. 22, n. 3, p. 523-581, 2007.

PARDO, I.; VARELA, C. La fecundidad bajo el reemplazo y las políticas familiares en América Latina y el Caribe: ¿qué puede aprenderse de la experiencia europea? Revista Brasileira de Estudos de População, v. 30, n. 2, p. 503-518, 2013.

PELLEGRINO, A. La población de Uruguay. Breve caracterización demográfica, Montevideo: UNFPA, 2010.

RALLU, L.; TOULEMON, L. Period fertility measures. The construction of different indices and their application to France, 1946-89. Population. An English Selection, n. 6, p. 59-94, 1994.

RIOS-NETO, E. L. G.; GUIMARÃES, R. R. de M. The educational gradient of low fertility in Latin America. En: ANNUAL MEETING OF POPULATION ASSOCIATION OF AMERICA. Anais... Boston, MA: PAA, 2014.

RODRÍGUE-VIGNOLI, J.; CAVENAGHI, S. Adolescent and youth fertility and social inequality in Latin America and the Caribbean: what role has education played? Genus, v. 70, n. 1, p. 1-25, 2014.

ROSERO-BIXBY, L.; CASTRO-MARTÍN, T.; MARTÍN-GARCÍA, T. Is Latin America starting to retreat from early and universal childbearing? Demographic Research, v. 20, n. 9, p. 169-194, 2009.

RYDER, N. B. Problems of trend determination during a transition in fertility. Milbank Memorial Fund Quarterly, n. 34, p. 5-21, 1956.

The process of demographic translation. Demography, n. 1, p. 74-82, 1964.

SOBOTKA, T.; LUTZ, W. Misleading policy messages derived from the period TFR: should we stop using it? Comparative Population Studies, v. 35, n. 3, p. 637-664, 2011.

SOBOTKA, T. Postponement of childbearing and low fertility in Europe. Tese (Doutorado) University of Groningen, Amsterdam, Dutch University Press, 2004.

SOBOTKA, T.; ZEMAN, K.; LESTHAEGHE, R.; FREJKA, T. Postponement and recuperation in cohort fertility: new analytical and projection methods and their application. Vienna Institute of Demography (VID) of the Austrian Academy of Sciences, 2011 (European Demographic Research Paper, n. 2-11).

SULLIVAN, R. The age pattern of first-birth rates among U.S. women: the bimodal 1990s. Demography, v. 42, n. 2, p. 259-273, 2005.

VARELA, C.; FOSTIK, A.; POLLERO, R. La fecundidad: evolución y diferenciales en el comportamiento reproductivo. En: VARELA, C. (Coord.). Demografía de una sociedad en transición: la población uruguaya a inicios del siglo XX. Montevideo: Trilce, 2008

VARELA, C.; PARDO, I. La heterogeneidad del comportamiento reproductivo: fecundidad y necesidades básicas insatisfechas. En: CALVO, J. J. (Coord.). Las necesidades básicas insatisfechas a partir de los Censos 2011. Montevideo: Trilce, 2013. 


\section{Sobre los autores}

Wanda Cabella es antropóloga y doctora en Demografía. Es Coordinadora del Programa de Población de la Facultad de Ciencias Sociales de la Universidad de la República, Uruguay.

Ignacio Pardo es sociólogo, magíster y doctor en Sociología. Es investigador y docente del Programa de Población de la Facultad de Ciencias Sociales de la Universidad de la República, Uruguay.

\section{Dirección para correspondencia}

Unidad Multidisciplinaria, Facultad de Ciencias Sociales

Constituyente $1502,4^{\circ}$ piso

11300 - Montevideo, Uruguay

\section{Resumo}

Chegou a hora de usar indicadores ajustados para estudar a fecundidade na América Latina?

0 artigo discute a pertinência do uso dos chamados indicadores refinados da fecundidade para avaliar o nível de fecundidade dos países latino-americanos. A maior vantagem desses indicadores é a sua capacidade de identificar quanto da mudança na taxa total de fecundidade deve-se à alteração da intensidade e quanto à alteração do calendário da fecundidade. Os países de fecundidade muito baixa estão finalizando um processo de postergação da maternidade (posponement transtion) que só conseguiu ser adequadamente descrito a partir do surgimento dos indicadores ajustados pelo efeito tempo. Embora na América Latina existam sinais de que alguns países começam a processar a postergação da maternidade, ainda é muito limitada a capacidade dos países da região para elaborar indicadores ajustados e, certamente, é quase inexistente a discussão sobre as suas vantagens, desvantagens e potencialidades. Com o objetivo de sistematizar a acumulação sobre esta questão e estimular o debate, apresentase as principais características das medidas ajustadas e discute quais os indicadores mais adequados para avaliar as tendências da fecundidade na região e como podem ser difundidos de modo a evitar equívocos em sua interpretação. A principal conclusão é que, para avançar hoje na análise da fecundidade latino-americana, o mais urgente a curto prazo é contar com indicadores de intensidade e calendário segundo ordem de nascimento, mais do que com indicadores sintéticos ajustados por tempo.

Palavras-chave: Indicadores ajustados de fecundidade; Postergação da maternidade. América Latina. Uruguai.

\section{Abstract}

Is it time to use more refined indicators to study fertility in Latin America?

The article discusses the appropriateness of refined fertility indicators to evaluate fertility level in Latin American countries. The main advantage of refined indicators is their ability to quantify the proportion of fertility change that is attributable to changes in the quantum of fertility and 
the proportion that is attributable to changes in the calendar. Lowest-low fertility countries are completing a process of fertility postponement that could only be accurately described when such refined indicators came into use. Despite indications that fertility postponement started in some Latin-American countries, the ability to produce refined estimators for the region is very limited, and a discussion on their advantages, disadvantages and potential is needed. The main properties of these measures are discussed, also which are the ones most appropriate to analyze fertility trends in the region, and how to avoid misleading interpretations. Our main conclusion is that, in the short term, it is more urgent to estimate tempo and quantum indicators by birth order, rather than estimating refined synthetic measures.

Keywords: Adjusted fertility indicators. Postponement transition. Latin America. Uruguay

Recebido para publicação em 13/05/2016 Recomendado para publicação em 19/08/2016 Aceito para publicação em 30/11/2016 